\title{
Natural Products Phenols as Novel Antithrombotic Agents
}

\author{
Wenjing Zhou and Ruihua Guo ${ }^{*, a, b, c}$ \\ ${ }^{a}$ College of Food Science and Technology, Shanghai Ocean University, Shanghai 201306, China \\ ${ }^{b}$ Shanghai Engineering Research Center of Aquatic-Product Processing \& Preservation, \\ Shanghai 201306, China \\ ${ }^{c}$ National Experimental Teaching Demonstration Center for Food Science and Engineering, \\ Shanghai Ocean University, Shanghai 201306, China
}

Email: rhguo@shou.edu.cn (R. G.)

\begin{abstract}
Thrombosis owns the highest incidence, and it is a serious threat to human health. Currently, the clinical antithrombotic drugs suffer from several drawbacks, causing allergic reactions or complications, etc. In recent years, small-molecule compounds isolated from natural products with antithrombotic activities in the field drug research on thrombus disease. In this review, we will focus on representative naturally occurring small molecule phenols with antithrombotic potency and potential for future therapeutic regimens to combat thrombosis disease.
\end{abstract}

Keywords phenols, thrombosis, antithrombotic activities, natural products, aglycon

\section{Introduction}

Thrombus is a severe health problem all over the world, especially in developing countries. It reduces the average life expectancy and changes the living habits. ${ }^{[1,2]}$ The current therapies (Heparin (ordinary heparin, low molecular weight heparin) and fondaparinux, and oral adjusted-dose vitamin $\mathrm{K}$ antagonists, ${ }^{[3]}$ aspirin, adenosine monophosphate receptor antagonist and platelet membrane glycoprotein antibody antagonist) are effective for the treatment of thrombosis patients clinically, however, they still remain unsatisfactory, ${ }^{[3-12,13-15]}$ Therefore, researchers are searching for small molecules with antithrombotic activities. ${ }^{[16-25]}$

Natural products are divided into diverse chemical compounds isolated from varied biological sources such as plants, animals, marine organisms, microorganisms, minerals, and organic matters. ${ }^{[26-29]}$ In recent years, the research on natural products becomes a hot issue in drug research. The drug discovery from natural products possess several advantages such as timeliness, faster and better methods for bioassay screening, and compound isolation. ${ }^{[30]}$ Currently, naturally occurring phenols with antithrombotic activities have been reported in the recent literature or patents, which would provide many clues to find the novel agents. This progress holds a considerable promise to the development of alternative, highly effective and secure therapeutics agents and ultimately the eradication of thrombosis disease. In this paper, we reviewed phenols from natural products with antithrombotic activities.

\section{Phenols}

Phenol compounds exist widely in natural products. Because of the strong antioxidant activity, ${ }^{[31]}$ phenols can delay tumor occurrence and inhibit the formation of tumor. ${ }^{[32]}$ It possesses anti-platelet activities. ${ }^{[33]}$

\section{Phenolic aglycon}

In 1997, a phenolic aglycom, resveratrol 3-O- $\beta$ - $D$-glucopyranoside 1 (Figure 1), was isolated from the seeds of Erythrophleum lasianthum (Caesalpinioidae, Leguminosae), a South African plant. ${ }^{[34]}$ The test results in vitro on human platelet-rich plasma demonstrated that resveratrol possesses strong inhibition on platelet aggregation induced by collage $\left(\mathrm{IC}_{50}=69 \mu \mathrm{M}\right)$, adrenalin $\left(\mathrm{IC}_{50}=102 \mu \mathrm{M}\right)$, arachidonic acid $\left(\mathrm{IC}_{50}=149 \mu \mathrm{M}\right)$ and $\mathrm{ADP}\left(\mathrm{IC}_{50}=218 \mu \mathrm{M}\right)$.<smiles>OCC1OC(Oc2cc(O)cc(C=Cc3ccc(O)cc3)c2)C(O)C(O)C1O</smiles>

Figure 1 The structures of compound 1.

\section{$m$-Hydroquinone}

Six hydroquinone constituents, resveratrol $(2),(+)$-catechin (3), (-)-catechin (4), (+)-epicatechin (5), (-)-epicatechin (6) and resorcinol (7), were isolated from the red wine in 2004 (Figure 2). ${ }^{[35,36]}$ All $m$-hydroquinone compounds had significant inhibition on peroxidase and cyclooxygenase reactions of COX-1. COX-1 exhibited a mechanism for antiplatelet agents. Compound $\mathbf{5}$ owns the strongest inhibition on peroxidase and cyclooxygenase reactions of COX-1 $\left(\mathrm{IC}_{50}=2.0\right.$ and $\left.12.2 \mu \mathrm{M}\right)$. Hence, they offer a wider pattern on the studies of antiplatelet field.

\section{Maltol}

In 2007, maltol 3-O- $\beta$-glucopyranoside 8 was isolated from Bark of Evodiopanax innovans (Araliaceae). ${ }^{[37]}$ The results indicated that it inhibited $50 \%$ platelet aggregation induced by collage, ADP, and thrombin. As a result, maltol 3-O- $\beta$-glucopyranoside is a significant bioactive substance. 
<smiles>Oc1ccc(/C=C/c2ccc(O)c(O)c2)cc1</smiles><smiles>Oc1cccc(O)c1</smiles>

Figure 2 The structures of compound 2-7.

\section{Polyphenols}

Seven polyphenols, vitisinols C 9, vitisinols D 10, (-)-viniferal $11,{ }^{[38]}$ ampelopsin C $12,,^{[39]}$ miyabenol A 13, ${ }^{[40]}$ $(+)$-vitisin A 14, ${ }^{[41]}$ and (+)-vitisin C $15^{[72]}$ were isolated from the dried roots of Vitis thunbergii in 2004 (Figure 3). ${ }^{[42]}$ All of the compounds had inhibitions on the platelet aggregation induced by $\mathrm{AA}$ and 9,11 -dideoxy-11R,9R-epoxy-methanoprostaglandin $\mathrm{F}_{2 a}$ (U46619, $\mathrm{TXA}_{2}$ analogous). Huang et al. revealed that $(+)$-vitisin $C 15$ had the strongest inhibition on the platelet aggregation induced by $\mathrm{AA}\left(\mathrm{IC}_{50}=5.7 \mu \mathrm{M}\right)$, and (-)-viniferal 11 had the strongest inhibition on the platelet aggregation induced by U46619 $\left(\mathrm{IC}_{50}=3.1 \mu \mathrm{M}\right)$.

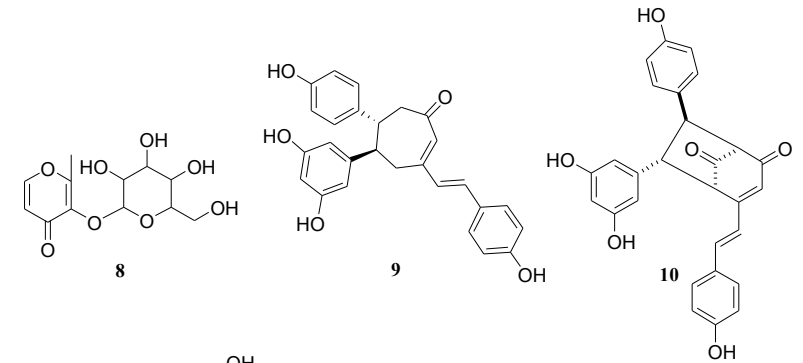

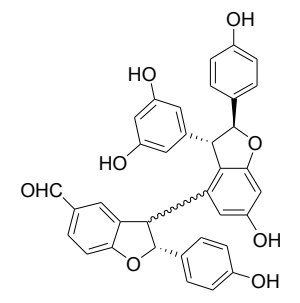

11

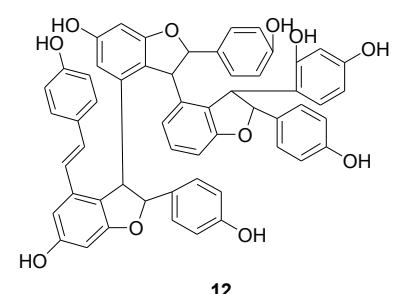

12

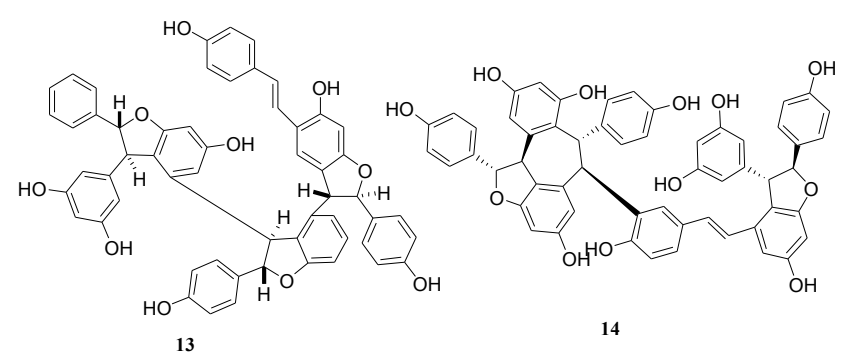

Figure 3 The structures of compound 8-14.

Six tannin compounds-polyphenol, Corilagin 16, penta- $O-$ galloyl- $\beta$-glucoside $17,{ }^{[43]}$ pedunculagin $18,^{[44]}$ tellimagrandin II $19,^{[44]}$ casuariin $20^{[44,45]}$ and 5-desgalloylstachyurin $21^{[46]}$ were naturally occurred (Figure 4). Corilagin 16 was isolated from Phyllanthus urinaria. It was found to be a potent fibrinolytic inhibitor $^{[47]}$ in 2003. Corilagin decreased PAI-1 activity $^{[48]}$ and enhanced t-PA activity both in vitro and in vivo $\left(\mathrm{IC}_{50}=39.7\right.$ $\mathrm{mg} / \mathrm{kg}) .{ }^{[49]}$ Other five compounds were isolated from the plant of Geum japonicum in 1998. ${ }^{[50]}$ Among of them, compound 17 showed the most significant effect on prolonging the clotting times of rabbit plasma. By their effects on proteolytic activity of thrombin tested, compound $\mathbf{1 9}$ showed the most potent activity $\left(\mathrm{IC}_{50}=0.070 \mu \mathrm{M}\right)$

\section{Phenolic acids}

Tanshen is listed in the Chinese Pharmacopoeia and largely used in Chinese traditional treatment. ${ }^{[51]}$ Few phenolic acids are isolated from Tanshen with anticoagulant and antiplatelet aggregation activities. ${ }^{[52]}$ Salvianolic acid A 22 exhibited strong anticoagulant activities. ${ }^{[53]}$ Fan et al. ${ }^{[54]}$ revealed that Salvianolic acid A 22 had no effect coagulation parameter in rats. It was approved that salvianolic acid A 22 could inhibit platelet aggregation induced by ADP without affecting coagulation system. In the human platelet aggregation assay, salvianolic acid A 22 had a potent inhibition on platelet aggregation induced by ADP with $\mathrm{IC}_{50}$ of $38.6 \mu \mathrm{g} / \mathrm{mL}$.
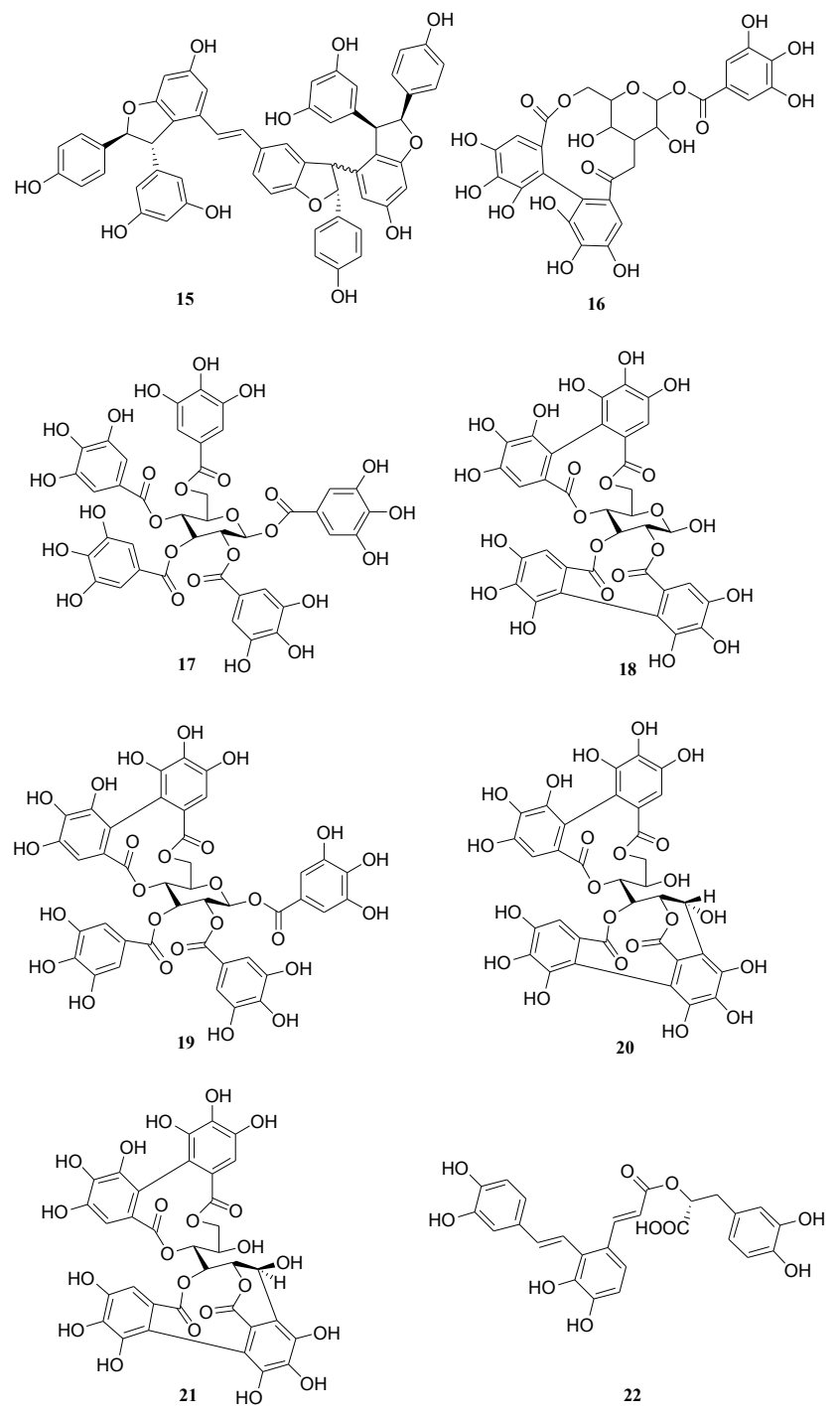

Figure 4 The structures of compound 15-22.

Another phenolic acid, Rosmarinic acid, showed a mild antithrombotic effect. ${ }^{[55]}$ In 1993, Zou et al. ${ }^{[56]}$ revealed that Rosmarinic acid 23 could inhibit the thrombosis by $41.9 \%$ and $54.8 \%$ on the concentration of the dosages of 50 and 100 
$\mathrm{mg} / \mathrm{kg}$. Meanwhile, it could also inhibit the platelet aggregation induced by collage $30.4 \%$ and $46.4 \%$ on the concentration of the dosages of 100 and $150 \mathrm{mg} / \mathrm{kg}$.

\section{Phloroglucinols}

Three phloroglucinol dimers, sideroxylonals A (24), sideroxylonal B (25) and Sideroxylonal C (26), were isolated from the flowers of Eucalyptus albens ${ }^{[57]}$ in 1998 (Figure 5). The sideroxylonals A-C (24-26) test showed the inhibition of PAI-1 $\left(\mathrm{IC}_{50}=3.3,5.3\right.$ and $4.7 \mu \mathrm{M}$, respectively). Other phloroglucinols with PAI-1 acitivities, euglobal IA2 (27), ${ }^{[57]}$ euglobal IIC (28) ${ }^{[58]}$ and robustadial $A(29)^{[59]}\left(\mathrm{IC}_{50}=138,700\right.$ and $152 \mu \mathrm{M}$, respectively), were also isolated.

\section{Others}

There were other works on phenol compounds. In 1994, two phenol compounds, moscatilin $\mathbf{3 0}$ and moscatin $\mathbf{3 1}$, were isolated from the stem of Dendrobium Idigesii, ${ }^{[60]}$ exhibiting strong inhibition of platelet aggregations. Compound 29 was acetylated with $\mathrm{Ac}_{2} \mathrm{O}$ to achieve compound 32. Compounds 30-32 showed the strong inhibition of platelet aggregations induced by $A A\left(\mathrm{IC}_{50}=61.8,37.2\right.$ and $11.2 \mu \mathrm{M}$, respectively). Compound 32 had a stronger activity than the other two compounds. Therefore, the natural products can act as good pro-drugs for inhibition of platelet aggregations. In 1995, a novel phenolic imperanene $\mathbf{3 3}$ was isolated from Imperata cylindrica $^{[61]}$ and it could inhibit at $6 \times 10^{-4} \mathrm{M}$ against rabbit platelet aggregation induced by thrombin.
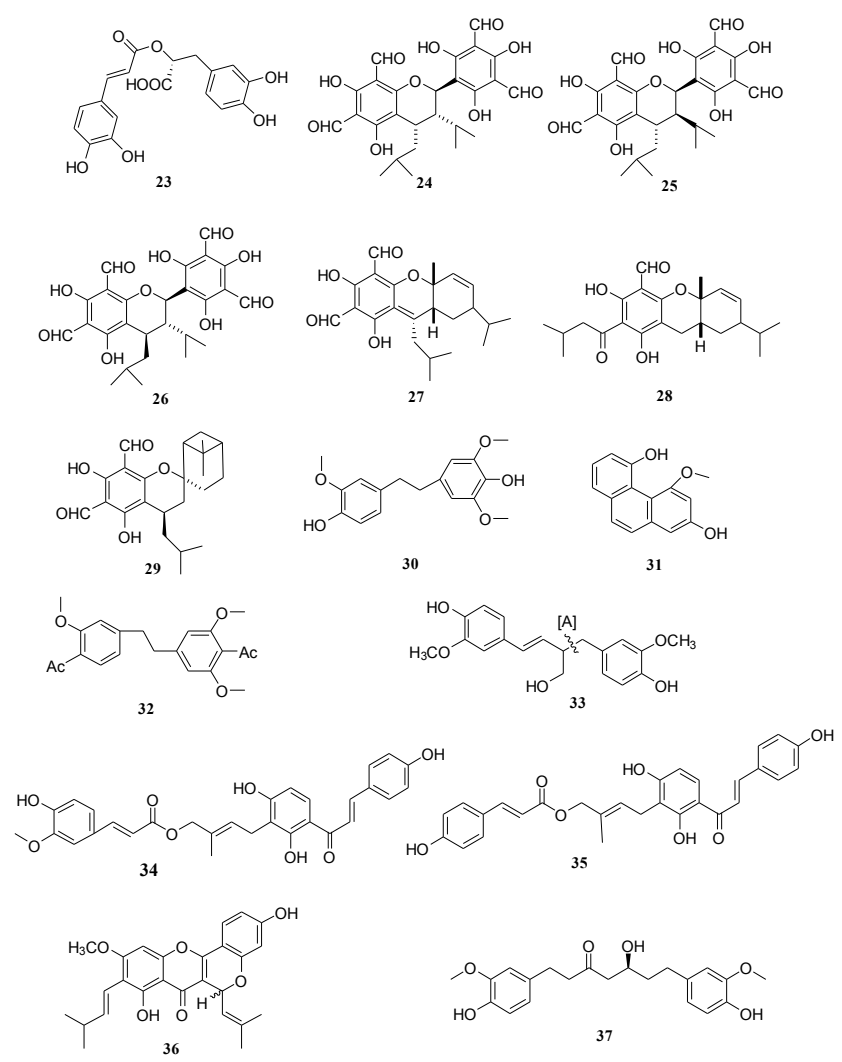

Figure 5 The structures of compound 23-37.

In 2002, few phenols were isolated including gemichalcone A (34), gemichalcone B (35) and cycloartocarpin (36). ${ }^{[62]}$ Compound 34 exhibited complete inhibition of platelet aggregation induced by $\mathrm{AA}$. Compound 35 also showed significant antiplatelet aggregation activity. Meanwhile, compound 36 showed the inhibition toward $A A$ and collagen completely. Dong et al. ${ }^{[96]}$ reported a curcumin derivative isolated from the root of gingers, hexahydrocurcumin (37), (5S)-hydroxy-1,7-bis(4-hydroxy-3-methoxyphenyl) heptan-3one. The platelet-rich human blood was tested to declare the antiplatelet aggregation. Hexahydrocurcumin was indicated to be a potent antiplatelet aggregation agent by experiment results.

\section{Conclusions}

This review summarized the small-molecule phenols antithrombotic active inhibitors. Phenols were characterized in phenolic aglycon, $m$-hydroquinone, maltol, polyphenols, phnolic acids, phloroglucinols. Their structural characteristics and antithrombotic properties (antiplatelet and anticoagulant therapies) were mainly discussed, which will provide valuable information for understanding the progress of antithrombotic activity investigation and developing new antithrombotic therapeutic agents.

Presently, due to drug high rate of bleeding, short half-life, and allergic reactions side-effects of current antithrombotic drugs, the search for novel drugs is mandatory. Therefore, many researchers pay more attention to small-molecule derived from natural resources.

Because of structural specificity, high activity and low toxicity of the natural products, they have been used as lead compounds for antithrombotic agents. Undoubtedly, natural products need to be investigated in more detail to obtain mechanistically and structurally novel and more potent derivatives and to explore their potential as novel adjuncts to established thrombosis disease.

\section{Acknowledgement}

The work was supported by the National Natural Science Foundation of China (No. 81502955), the Doctoral Scientific Research Foundation of Shanghai Ocean University (No. A2030214300077), the Young Teachers Training Program of Shanghai (No. A12056160002), the Plan of Innovation Action in Shanghai (No. 14431906000), and the Project Funded by Jiangsu Key Laboratory of Marine Pharmaceutical Compound Screening.

\section{References}

[1] Nicholson, C. D.; Lensing, A. W. Expert Opin. Investig. Drugs 2001, 10, 785.

[2] Lee, Y. K.; Player, M. R. Med. Res. Rev. 2011, 31, 202.

[3] Quinlan, D. J.; Eriksson, B. I. Best Pract. Res. Clin. Haematol. 2013, 26, 171.

[4] Wei, X.; Yin, R. X.; Cardiology, D. O. Med. Recapitulate 2014, $20,4520$.

[5] Xavier, A. R.; Siddiqui, A. M.; Kirmani, J. F.; Hanel, R. A.; Yahia, A. M.; Qureshi, A. I. CNS Drugs 2003, 17, 213.

[6] Gross, P. L.; Weitz, J. I. Clin. Pharmacol. Ther. 2009, 86, 139.

[7] Johannes, R.; Katus, H. A. Expert Opin. Investig. Drugs 2003, $12,781$.

[8] Hsieh, F.; Hsu, C.; Huang, H.; Shiau, C. FEBS J. 2005, 272, 407.

[9] Balasubramaniam, K.; Viswanathan, G. N.; Marshall, S. M.; Zaman, A. G. Cardiol. Res. Pract. 2012, 2012, 1.

[10] Lees, K. R.; Bluhmki, E.; Kummer, R. V.; Brott, T. G.; Toni, D.; Grotta, J. C.; Albers, G. W.; Kaste, M.; Marler, J. R.; Hamilton, S. A. Lancet 2010, 375, 1695.

[11] Peter, S.; Wardlaw, J. M.; Lindley, R. I.; Martin, D.; Geoff, C.; Gordon, M.; Karen, I.; Graham, V.; Anna, C.; Adam, K. Lancet 
2012, 379, 2352

[12] Wardlaw, J. M.; Murray, V.; Berge, E.; Zoppo, G.; Sandercock, P.; Lindley, R. L.; Cohen, G. Lancet 2012, 379, 2364.

[13] Guo, R. H.; Zhang, Y. T.; Duan, D.; Fu, Q.; Zhang, X. Y.; Yu, X. W.; Wang. S. J.; Bao, B.; Wu, W. H. Chin. J. Chem. 2016, 34, 1194.

[14] Wang, G.; Wu, W. H.; Zhu, Q. G.; Fu, S. Q.; Wang, X. Y.; Hong, S. T.; Guo, R. H.; Bao, B. Chin. J. Chem. 2015, 33, 1089.

[15] Guo, R. H.; Duan, D.; Zhang, Y. T.; Fang, Y. W.; Wu, W. H. Med. Res. 2017, 1, 12.

[16] Friederich, P. W.; Levi, M.; Biemond, B. J.; Charlton, P.; Templeton, D.; Zonneveld, A. J., Van; Bevan, P.; Pannekoek, H.; Cate, J. W. Circulation 1997, 96, 916.

[17] Qiao, J. X.; Wang, T. C.; Réjean, R.; Carl, T.; Alexandre, L. H.; Schumacher, W. A.; Spronk, S. A.; Sheldon, H.; Gilles, B.; John, L. J. Med. Chem. 2013, 56, 9275.

[18] Massimo, C.; Savitri, K.; Das, C. M.; Reuben, J. M.; William, S.; Jamileh, N.; Frederick, B.; Gascoyne, P. R. Circ. Res. 2012, $111,920$.

[19] Louisa, D.; Sim, D. S.; Dilks, J. R.; Price, B.; Sarah, B.; Denker, B. M.; Georgios, K.; Athan, K.; Robert, F. Proc. Natl. Acad. Sci. 2011, 108, 2951.

[20] Bullingham, A.; Strunin, L. Brit. J. Anaesth. 2009, 339, 622.

[21] Abboud, M.; Needle, S.; Burns-Kurtis, C.; Valocik, R. P.; Amour, A.; Chan, C.; Brown, D.; Chaudry, L.; Zhou, P.; Patikis, A. J. Cardiovasc. Pharm. 2008, 52, 66.

[22] Eriksson, B. I.; Dahl, O. E.; Lassen, M. R.; Ward, D. P.; Rothlein, R.; Davis, G.; Turpie, A. G. G. J. Throm. Haemost. 2008, 6, 457.

[23] Kolesnikov, A.; Rai, R.; Mordenti, J.; Liu, L.; Torkelson, S.; Shrader, W.; Leahy, E.; Hu, H.; Gjerstad, E.; Janc, J. Bioorg. Med. Chem. Lett. 2006, 16, 2243.

[24] Srivastava, S.; Goswami, L. N.; Dikshit, D. K. Med. Res. Rev. 2005, 25, 66

[25] Rai, R.; Kolesnikov, A.; Yong, L.; Young, W. B.; Leahy, E.; Sprengeler, P. A.; Verner, E.; Shrader, W. D.; Burgess, H. J.; Sangalang, J. C. Bioorg. Med. Chem. Lett. 2001, 11, 1797.

[26] Grover, J. K.; Yadav, S.; Vats, V. J. Ethnopharmacol. 2002, 81, 81.

[27] Hansen, E.; Andersen, J. H. Curr. Pharm. Biotechnol. 2015, 17, 71.

[28] Orlikova, B.; Legrand, N.; Panning, J.; Dicato, M.; Diederich, M. Cancer Treat. Res. 2014, 159, 123.

[29] Sharma, S. B.; Gupta, R. Mini-Rev. Med. Chem. 2015, 15, 52.

[30] Tian, L.; Teng, X.; Zhong, C. Gen. Chem. 2015, 1, 22.

[31] Bravo, L. Nutr. Rev. 1998, 56, 317.

[32] Das, M.; Khan, W. A.; Asokan, P.; Bickers, D. R.; Mukhtar, H. Cancer Res. 1987, 47, 767.

[33] Pawlaczyk, I.; Czerchawski, L.; Kuliczkowski, W.; Karolko, B.; Pilecki, W.; Witkiewicz, W.; Gancarz, R. Thromb. Res. 2011, $127,328$.

[34] Rocca, G. D.; Pugliese, F.; Antonini, M.; Coccia, C.; Pompei, L.; Venuta, F.; Rendina, E.A.; Ricci, C. J. Nat. Prod. 1997, 60, 1082.

[35] Szewczuk, L. M.; Penning, T. M. J. Nat. Prod. 2004, 67, 1777.
[36] Pace A. C. R.; Hahn, S.; Diamandis, E. P.; Soleas, G.; Goldberg, D. M. Clin. Chim. Acta 1995, 235, 207.

[37] Tsuchiya, H.; Tanaka, T.; Nagayama, M.; Oyama, M.; linuma, M. Nat. Prod. Commun. 2008, 3, 809 .

[38] Ito, J.; Niwa, M. Tetrahedron 1996, 52, 9991.

[39] Li, W. W.; Ding, L. S.; Li, B. G.; Chen, Y. Z. Phytochemistry 1996, 42, 1163

[40] Kawabata, J.; Mishima, M.; Kurihara, H.; Mizutani, J. Phytochemistry 1995, 40, 1507.

[41] Ito, J.; Gobaru, K.; Shimamura, T.; Niwa, M.; Takaya, Y.; Oshima, Y. Tetrahedron 1998, 54, 6651.

[42] Yu, L. H.; Wei, J. T.; Chien, C. S.; Chien, C. C. J. Nat. Prod. 2005, 68, 217

[43] Yoshida, T.; Chen, L.; Shingu, T.; Okuda, T. Chem. Pharm. Bull. 1988, 36, 2940.

[44] Okuda, T.; Yoshida, T.; Ashida, M.; Yazaki, K. J. Chem. Soc. Perkin Trans. 1983, 1765.

[45] Nonaka, G. I.; Sakai, T.; Tanaka, T.; Mihashi, K.; Nishioka, I. Pharm. Bull. 1990, 38, 2151

[46] Lee, S. H.; Tanaka, T.; Nonaka, G. I.; Nishioka, I. Phytochemisry 1990, 29, 3621.

[47] Dong, H.; Chen, S. X.; Kini, R. M.; Xu, H. X. J. Nat. Prod. 1998, $61,1356$.

[48] Jia, T.; Wei, D. Y.; Lin, C.; Fang, J. I.; Chao, Z. X.; Qiang, S. Z. J. Kunming Med. Univ. 2009, 30, 14.

[49] Shen, Z. Q.; Chen, P.; Shen, J. Q.; Dong, Z. J.; Liu, J. K. Nat Prod. Res. Dev. 2003, 15, 441.

[50] Dong, S. X.; Kini, R. M.; Xu, H. X.; Dong, H.; Chen, S. X.; Xu, H. X. J. Nat. Prod. 1998, 61, 1356.

[51] Ouyang, X.; Takahashi, K.; Komatsu, K.; Nakamura, N.; Hattori, M.; Baba, A.; Azuma, J. Jap. J. Pharm. 2002, 87, 289.

[52] Wang, X.; Morrisnatschke, S. L.; Lee, K. H. Med. Res. Rev. 2007, 27, 133.

[53] Lian, N. L.; Rui, T.; Wei, M. C. Planta Med. 1984, 50, 227.

[54] Fan, H. Y.; Fu, F. H.; Yang, M. Y.; Xu, H.; Zhang, A. H.; Liu, K. Thromb. Res. 2010, 126, 17.

[55] Ren, W. J.; Kit, M. L.; Po, M. H.; Mak, T. C. W.; Kam, S. W.; Kwok, P. F. Curr. Med. Chem. 2005, 12, 237.

[56] Zou, Z. W. Acta Pharm. Sinica 1993, 28, 241.

[57] Neve, J.; Leone, P. A.; Carroll, A. R.; Moni, R. W.; Paczkowski, N. J.; Pierens, G. B.; Rquist, P.; Deinum, J.; Ehnebom, J.; Inghardt, T. J. Nat. Prod. 1999, 62, 324.

[58] Kozuka, M.; Sawada, T.; Kasahara, F.; Mizuta, E.; Amano, T.; Komiya, T.; Goto, M. Chem. Pharm. Bull. 1982, 30, 1952.

[59] Cheng, Q.; Snyder, J. K. J. Org. Chem. 1988, 53, 4562.

[60] Kang, J.; Cabral, C.; Kushner, L.; Salzman, E. W. Blood 1994, $57,1271$.

[61] Matsunaga, K.; Shibuya, M.; Ohizumi, Y. J. Nat. Prod. 1995, 58, 138

[62] Mei, I. C.; Jing, R. W.; Jih, P. W.; Che, M. T.; Chun, N. L. Planta Med. 2002, 68, 25.

Received March 8, 2018 Accepted March 22, 2018 